\title{
TURISMO E INMIGRANTES EXTRANJEROS DE ORIGEN COMUNITARIO: EL DESARROLLO DE EMPRESAS TURÍSTICAS EN EL LITORAL CATALÁN ${ }^{1}$
}

\author{
Raúl LARDIÉS \\ Departamento de Geografía y Ordenación del Territorio \\ Universidad de Zaragoza
}

\begin{abstract}
Resumen: En este artículo se examina el desarrollo de empresas turísticas por parte de inmigrantes extranjeros en el litoral de Cataluña (España). Gracias a la realización de 113 entrevistas a pequeños empresarios de turismo originarios de paises comunitarios durante 1996 y 1997, se han analizado diversos aspectos de tipo social y cultural referidos a la instalación de estas empresas - por ejemplo las motivaciones de los empresarios para el desplazamiento y la apertura de los negocios, sus cualificaciones, la experiencia profesional previa en turismo y las fuentes de capital para poner en marcha la empresa-. La conclusión es que tanto el desplazamiento de estos ciudadanos a Cataluña como la apertura de los negocios están motivados fundamentalmente por razones de tipo personal, familiar, climático y medioambiental, en lugar de por las estrictamente económicas o laborales. Los resultados obtenidos apuntan a que las teorías sobre migraciones internacionales aportan pocas explicaciones sobre estos flujos de inmigrantes.
\end{abstract}

Palabras clave: Inmigración, noreuropeo, Mediterráneo, Cataluña, turismo, empresario, empresa turística.

\begin{abstract}
This paper examines EU foreign entrepreneurs in the tourist industry in the coastal areas of Cataluña (Spain). Thanks to the completion of 113 interviews with foreign tourism entrepreneurs during 1996 and 1997, some cultural and social aspects of north-European citizens with small tourism businesses are analyzed —for instance
\end{abstract}

\footnotetext{
1 Este artículo recoge algunos resultados del trabajo desarrollado por el autor durante su estancia de dos años en el departamento de Geografía del King's College (Universidad de Londres) como becario predoctoral del programa Human Capital and Mobility de la Unión Europea. La elaboración de este artículo ha sido también posible gracias a la realización posterior de una estancia de investigación en el mismo departamento con motivo de la concesión de una beca del «Programa Europa de Estancias de Investigación» (CAI/DGA). El autor agradece al doctor Keith Hoggart del departamento de Geografía del King's College su ayuda durante el tiempo que duró la investigación.
} 
their business motivations, qualifications, previous experience in the tourism sector and sources of capital to start the business-. As with the reasons for moving to this region, firm formation and the manner in which these tourist businesses are run is found to be motivated less by work and economic reasons than by lifestyle considerations. They must be seen as an issue of «consumption», instead of one of work and the production of services. The results suggest that international migration theory offers little to explain these migration inflows of European migrants with tourist businesses into the coastal areas of Cataluña.

Keywords: Immigration, north-European, Mediterranean, Cataluña, tourism, entrepreneur, tourism business.

\section{INTRODUCCIÓN}

Finalizadas las corrientes migratorias que durante décadas se dirigieron desde los países del sur de Europa a los del norte, la escena migratoria europea está dominada actualmente por nuevos flujos de población. Junto a los movimientos de refugiados desde el este al oeste europeo o los movimientos de trabajadores de alta cualificación, que parecen ser los numéricamente dominantes (SALT, 1996), los desplazamientos desde los países del norte de Europa a los del sur están produciendo una nueva distribución geográfica de las migraciones en Europa. Pero a diferencia de los flujos de décadas pasadas, muchos de los actuales no están motivados fundamentalmente por motivos de trabajo, sino de consumo, y ligados mayoritariamente a otros desplazamientos previos de carácter turístico (BULLER y HOGGART, 1994). En este artículo se analizan estos nuevos flujos, prestando atención a los inmigrantes comunitarios (en su mayoría noreuropeos) que han desarrollado pequeños negocios turísticos en el litoral catalán.

Los resultados de este artículo se basan en 113 entrevistas realizadas durante 1996 y 1997 a inmigrantes extranjeros de origen europeo que eran propietarios o regentaban pequeños negocios turísticos. La selección de Cataluña como zona de estudio responde al importante peso del sector turístico en la economía de la región, así como al gran número de residentes extranjeros originarios de países comunitarios y su fuerte concentración en el litoral mediterráneo (LARDIÉS y HOGGART, 1996). SHAW y WILLIAMS (1990) reconocen que la relación existente entre el turismo y las empresas del sector depende del tipo de desarrollo turístico producido. En tal caso, es sabido que España es el destino principal cada año de millones de turistas noreuropeos y que esa gran demanda noreuropea ha contribuido al desarrollo turístico de la zona mediterránea española (NAYLON, 1992). En particular, Cataluña ha sido una de las regiones en donde más se ha desarrollado este sector, y que mayor número de turistas noreuropeos recibe. Por tanto, un objetivo de este análisis es ver también si existe alguna relación entre los flujos turísticos dominantes y las pautas migratorias de estos inmigrantes extranjeros. 
La investigación en nuestro país ha profundizado muy poco en la relación existente entre la inmigración de países noreuropeos y su impacto en el desarrollo de empresas turísticas (LARDIÉS, 1999). Ello es debido a dos razones. La primera es que, desde el punto de vista de las migraciones de población, los flujos desde países del norte de Europa a los del sur son relativamente recientes y sus protagonistas pertenecen a unas nacionalidades que no han participado tradicionalmente en las grandes corrientes migratorias (ZLOTNIK, 1992). La segunda razón es que, desde el punto de vista del empresariado turístico, se han realizado muy pocos estudios sobre este aspecto, y mucho menos referidos a empresarios de origen extranjero. Sin embargo, esta parquedad de estudios en España contrasta con los trabajos más numerosos que se han realizado en algunos países europeos, la mayor parte de los cuales se refieren a empresarios turísticos de origen extranjero con negocios en grandes urbes, y pertenecientes casi siempre a minorías étnicas procedentes de países asiáticos, africanos o caribeños (WALDINGER et alt., 1990; WARD, 1991).

Los estudios sobre el turismo en España se han centrado mayoritariamente en aspectos como la oferta o demanda de determinados servicios, o en los flujos entre lugares de origen y destino (WILLIAMS y SHAW, 1988; ANTON CLAVÉ et alt., 1996). En cambio, los aspectos sociales y culturales del turismo referidos al empresariado y a la formación y funcionamiento de las empresas han estado muy descuidados como objeto de estudio. Mucho más, cuando ese empresariado es extranjero y se intenta analizar la formación de empresas en el contexto de la inmigración extranjera. Sobre este particular, algunas de las mejores referencias bibliográficas a escala europea las constituyen los trabajos realizados por $\mathrm{G}$. SHAW y A.M. WILLIAMS (SHAW y WILLIAMS, 1987; SHAW y WILLIAMS, 1988; WILLIAMS y SHAW, 1988; WILLIAMS et alt., 1989a; WILLIAMS et alt., 1989b), en donde se analizan desde un punto de vista sociocultural las diferentes vías para acceder al empresariado turístico en la región inglesa de Cornwall, aunque sólo teniendo en cuenta los empresarios de origen nacional.

\section{LA TERCIARIZACIÓN DE LA ECONOMÍA Y LAS OPORTUNIDADES LABORALES PARA LOS INMIGRANTES EXTRANJEROS}

Un hecho que refleja las transformaciones económicas producidas en los países europeos en las últimas décadas ha sido el crecimiento del empleo en el sector terciario (OECD, 1992). Estas transformaciones han supuesto, por un lado, el abandono del sistema de producción masivo caracterizado por la producción industrial en grandes empresas y, por otro, la adopción de formas de producción más flexibles y a menor escala. El resultado ha sido un trasvase importante de población activa desde el sector secundario al terciario, aglutinando este último la mayor parte de la población activa. Precisamente, es en países del sur de Europa donde mayor es la flexibilidad en la producción y donde las nuevas formas de organización del mercado de trabajo potencian la creación de «nichos» laborales para inmigrantes extranjeros, en relación también con prácticas más o menos marginales o ilegales (KING y RYBACZUK, 1993). 
Este crecimiento del empleo terciario está teniendo un claro beneficio en los inmigrantes extranjeros, y prueba de ello es la creciente participación de los trabajadores de origen extranjero en las economías de los países europeos durante la última década (OECD, 1994). El sector turístico es uno de los que mayor número de extranjeros ocupa y, por tanto, en este escenario es donde debemos contextualizar el fenómeno de las empresas turísticas puestas en marcha por algunos grupos de inmigrantes extranjeros en Cataluña. Se trata de trabajos que, en la práctica, pueden ser ocupados sin poseer una cualificación determinada ni una formación profesional especial, con lo cual la penetración se hace más fácil. Ello se traduce en la relativa facilidad para la apertura de un pequeño negocio en el sector de los servicios, y en particular en las ramas de la hostelería y la hotelería.

Al margen de las posibilidades que la situación económica general ofrece para los inmigrantes, la gran afluencia turística desde países europeos, y en especial los del norte, ha tenido grandes consecuencias para España y en especial para Cataluña. En concreto, por lo que se refiere a la atracción e instalación de inmigrantes noreuropeos en la costa mediterránea y la posterior apertura de un negocio turístico, esta gran afluencia de turismo noreuropeo ha hecho aumentar sustancialmente las oportunidades de empleo para los inmigrantes de esos países. Primero, porque gracias a las estancias turísticas España llega a convertirse en un destino conocido y familiar. Segundo, porque todo ese turismo supone un gran mercado para explotar, en el sentido de que una gran demanda de turismo extranjero ofrece el marco perfecto para el desarrollo de negocios por parte de inmigrantes extranjeros (GIBSON, 1992).

\section{EL ESTUDIO DE LOS EMPRESARIOS DE ORIGEN EXTRANJERO}

\section{Principales Fuentes y Metodología}

Entrando ya en el tema específico del análisis de los empresarios turísticos de origen extranjero y sus empresas, el principal problema que se plantea a la hora de llevar a cabo su estudio es la carestía de información estadística, tanto para su cuantificación como para conocer algunas de sus características socioeconómicas de los empresarios, incluida su distribución por sectores en la economía regional. Tal y como hemos explicado (LARDIÉS, 1999), esta falta de información es debida, principalmente, al escaso control que se tiene sobre esta población, una vez establecida la libertad para la circulación de personas y prestación de servicios en cualquier otro país miembro de la Unión Europea. Esta ha sido la principal causa de que estos inmigrantes se conviertan en un grupo inaccesible desde el punto de vista estadístico.

Por ejemplo, la falta de datos oficiales impide conocer no sólo el número de inmigrantes (empresarios) repartidos por tipos de negocios, sino también las evoluciones tempora- 
les del número de establecimientos en manos de estos inmigrantes. Tal dificultad, por tanto, ha hecho necesario acceder a fuentes de información como las Cámaras de Comercio, ayuntamientos o asociaciones de empresarios turísticos. Así, el acceso a los empresarios objeto de nuestro estudio se produjo principalmente gracias a los censos de actividades de las Cámaras de Comercio e Industria de la región, pudiendo obtener de ese modo una aproximación, aunque sesgada, tanto de su número como de su distribución geográfica y en las distintas ramas del sector turístico.

Aun con todo, fue necesario realizar entrevistas personales a los empresarios objeto de estudio, por lo que durante 1996 y 1997 se realizaron (en inglés, francés y español) 113 entrevistas a empresarios procedentes de alguno de los antiguos 12 países miembros de la CEE. El objetivo de estas entrevistas era conocer algunas de sus características (país de origen, motivaciones para emigrar u ocupaciones profesionales previas), como también de las empresas (fecha de instalación, fuentes de financiación, empleo generado y su temporalidad, etc.). Dada la diversidad de actividades que el sector turístico engloba y las muchas que tienen relación indirecta con el mismo, bajo la consideración de empresas turísticas se incluyeron las de las ramas de restauración y alojamiento, animación, negocios inmobiliarios, así como también los propietarios de pequeño comercio. En concreto, se entrevistó a propietarios de negocios de hostelería y restauración (44 restaurantes, 17 bares y 6 cafeterías/heladerías), locales de diversión (14 pubs/discotecas), hotelería (5 hoteles y una vivienda de turismo rural), de inmobiliarias y alquiler de apartamentos (2), y de pequeños comercios (7 de la rama de hogar y decoración, 3 de alimentación, 4 de papelería y librería, y otros 10 pertenecientes a otras ramas).

Los puntos donde se realizaron las entrevistas fueron elegidos en función de la concentración territorial de residentes extranjeros de origen comunitario, y gracias también a los listados de empresarios facilitados por las Cámaras de Comercio y Asociaciones de turismo. Por ello, la distribución geográfica de los empresarios entrevistados coincide a grandes rasgos con la de los residentes extranjeros procedentes de otros países comunitarios y ofrecida por el Censo de Población. Así, en la provincia de Gerona están concentrados en municipios costeros el $56 \%$ de esos residentes extranjeros, en la de Barcelona el $63,3 \%$ y en la de Tarragona el 73,1\% (el 64,1\% entre las tres provincias) (IEC, 1992). A la vista de esa distribución, para las entrevistas se siguió el mismo patrón, realizándose el $93,8 \%$ de ellas en núcleos costeros o a una distancia máxima de 20 kilómetros de la línea de costa. En concreto, dejando fuera del trabajo de campo la parte más septentrional de la Costa Brava, la mayoría de las entrevistas se realizaron en los núcleos de población localizados entre Palafrugell y Blanes, además de en Tarrasa y Sabadell, Sitges y los principales destinos turísticos de la Costa Dorada (entre Torredembarra y L'Ametlla de Mar).

\section{Establecimiento del negocio}

La primera característica importante obtenida de las entrevistas realizadas es la fecha de instalación de la empresa (de la primera de ellas, en el caso de que haya habido otras 
posteriores ligadas a cambios de residencia). Conocer este dato es importante por tres razones. Primero, porque permite saber si la fecha en que se instala la empresa es la misma que en la que se inmigra a España/Cataluña. En principio, son dos hechos que no tienen porqué coincidir, y así ocurre como veremos. Segundo, porque la fecha del establecimiento del negocio se puede relacionar con el momento de mayor o menor desarrollo turístico en la región, de modo que se puede ver si los años del boom de afluencia de turismo noreuropeo son los de mayor o menor apertura de negocios por parte de los inmigrantes de estos países. Tercero, porque la supresión de barreras institucionales entre los países comunitarios puede haber influido en el mayor o menor establecimiento de negocios por parte de inmigrantes procedentes de países comunitarios. Por tanto, conociendo las fechas de puesta en funcionamiento de los negocios, se podrá analizar la influencia de la libre circulación de personas en el espacio europeo en la mayor o menor apertura de negocios.

El abanico de fechas de instalación de estos negocios no es muy amplio (tabla 1). Casi la mitad fueron creados con posterioridad al año 1990 (48,7\%), y casi dos tercios $(63,7 \%)$ después de 1985. Tan sólo un 23\% de los negocios que en 1996-97 sobrevivían se pusieron en funcionamiento antes de 1980, mientras que los anteriores a 1970 son realmente escasos $(7,1 \%)$. El hecho de que las empresas empiecen a funcionar en esos años, no supone que sus propietarios se hayan establecido en el país en la misma fecha. Aunque normalmente el tiempo transcurrido entre el momento en que la persona fija su residencia en nuestro país y la apertura del negocio es escaso (el 51,3\% de los empresarios abrieron su negocio nada más establecer su residencia), en otras ocasiones los dos hechos no van asociados. De todos los entrevistados, es significativo que casi un 15\% tardó en poner en marcha su primer negocio seis años o más, mientras que un $8,9 \%$, esperaron entre dos y seis años. El hecho de que casi el $24 \%$ de los entonces inmigrantes extranjeros tardaran dos o más años en establecer su negocio apunta a la inseguridad y a la falta de una iniciativa profesional concreta en el momento de fijar su residencia en España, y al hecho de no tener demasiado claro el tipo de actividad económica a la que deseaban dedicarse.

Tabla 1. Fecha de apertura de los negocios

\begin{tabular}{|c|c|c|}
\hline Fecha de apertura & Nimero & $\%$ \\
\hline $1969 \mathrm{y}$ antes & 8 & 7,1 \\
\hline 1970 a 1974 & 10 & 8,8 \\
\hline 1975 a 1979 & 8 & 7,1 \\
\hline 1980 a 1984 & 15 & 13,3 \\
\hline 1985 a 1989 & 17 & 15,0 \\
\hline 1990 a 1994 & 33 & 29,2 \\
\hline 1995 y después & 22 & 19,4 \\
\hline TOTAL & 113 & 100,0 \\
\hline
\end{tabular}


Aunque las cifras apuntan claramente a la relativa modernidad de las empresas, vamos a relacionar las distintas fechas de apertura de los negocios con los años de mayor y menor crecimiento del turismo extranjero en Cataluña. Tal y como SHAW y WILLIAMS (1998) señalan, es en las primeras etapas del desarrollo turístico local cuando la industria del sector tiende a estar escasamente evolucionada. En esa fase es normal la falta de preparación y experiencia profesional entre los trabajadores locales en las principales ramas relacionadas con la economía turística (hotelería, hostelería y restauración, alimentación, etc.). En consecuencia, en ese momento es en el que se produce la mayor penetración de trabajadores extranjeros para desarrollar iniciativas empresariales en el sector, proveyendo de esa manera práctica conocimientos entre los empresarios locales.

Sin embargo, este comportamiento detectado en muchos casos, no es aplicable al nuestro. La reciente apertura de estos negocios en Cataluña es el reflejo de que la gran emergencia de estas empresas no se produjo durante las etapas iniciales del desarrollo del turismo masivo en la costa catalana llevada a cabo entre los años 60 y 70 . O si lo fue, las empresas que han sobrevivido desde esos años son realmente escasas. Al contrario, las cifras muestran que la mayoría de los negocios que hoy sobreviven han sido creados posteriormente al boom turístico. En cualquier caso, su creación parece que responde a la existencia de una demanda extranjera, que en numerosas ocasiones constituye la principal clientela. Eso apoya la idea, por tanto, de que más que contribuir a aumentar la demanda extranjera en ramas y tipos de negocios diferentes, a lo que este empresariado turístico extranjero contribuye es a consolidar la clientela extranjera en los negocios tradicionales como los de hostelería, restauración y entretenimiento (pubs y discotecas). En principio, lo que el análisis de la operatividad temporal de estas empresas sugiere (y antes de conocer las razones de los empresarios para establecer el negocio) es que las que comenzaron a funcionar en la década de los años 60 , más que el resultado de una «colonización extranjera» de empresarios destinados a ocupar esos vacíos laborales mediante la aportación de profesionalidad y experiencia, responden a cuestiones personales, aunque relacionadas, a la vez, con las nuevas oportunidades laborales de satisfacer a los clientes extranjeros que en ese momento comenzaban a llegar masivamente.

No obstante, es difícil explicar exactamente porqué el mayor número de empresas ha sido creado en los últimos años (desde 1990). Por un lado hay que tener en cuenta que las entrevistas se han realizado a las únicas supervivientes, por lo que no sabemos qué proporción de todas las instaladas en los años 60 se han cerrado desde entonces. Por ello, intentando hallar una explicación para esta explosión reciente de empresas, es necesario tener en cuenta otros factores además de los anteriores.

Uno de los que más puede haber influido en la mayor o menor fijación de estas empresas ha sido la supresión de barreras institucionales entre los países de la Unión Europea y la libertad de los ciudadanos de los Estados miembros para residir y trabajar en cualquier otro país de la Unión. Sin embargo, la falta de información relativa al establecimiento de negocios por parte de estos ciudadanos comunitarios dificulta ese análisis, aunque siempre se pueden establecer similitudes entre cómo han evolucionado los flujos migratorios 
de población entre los países europeos a raíz de la supresión de las citadas restricciones (SIMON, 1993) y la mayor o menor creación de negocios turísticos por ciudadanos extranjeros. Según los datos obtenidos de las entrevistas, el hecho de que sólo el 10,6\% de los entrevistados opinen que no tuvieron problemas en la apertura del negocio por el hecho de ser originarios de otro país comunitario, no es realmente muy significativo. No haber tenido problemas por pertenecer a otro país miembro de la Unión, no quiere decir que la libertad de movimiento e instalación haya influido en algo a la hora de tomar la decisión de emigrar y establecer posteriormente el negocio. Además, cuando se les preguntó por las motivaciones para establecerlo en Cataluña, ningún empresario señaló que la libre circulación de personas entre los Estados miembros y la facilidad para el establecimiento de una empresa había sido decisivo o importante. Ello permite pensar que entre las motivaciones para que la mayoría de empresas se hayan establecido durante la última década no destacan precisamente las ligadas a la reciente libertad de movimiento y a la facilidad para prestar de servicios en cualquier otro país comunitario.

\section{Origen geográfico de los empresarios}

Una característica importante de este grupo heterogéneo de empresarios es su origen geográfico (tabla 2). Dentro de la variedad de países a los que pertenecen los empresarios, destaca el predominio de los procedentes de países del norte de Europa (incluyendo Francia, el 79,7\%) sobre los sureuropeos (sólo representados los italianos, con un 20,3\%). La mayoría de empresarios pertenecen a un reducido número de nacionalidades, con los alemanes a la cabeza $(23,1 \%)$, seguidos de los italianos $(20,3 \%)$, los franceses $(17,7 \%)$ y los británicos $(17,7 \%)$, mientras que la otra nacionalidad que por el número de sus miembros

Tabla 2. Origen de los empresarios

\begin{tabular}{|lcr|}
\hline Pais de origen & Nimero & $\%$ \\
\hline Alemania & 26 & 23,1 \\
Bélgica & 5 & 4,4 \\
Dinamarca & 1 & 0,9 \\
Francia & 20 & 17,7 \\
Grecia & 0 & 0,0 \\
Países Bajos & 18 & 15,9 \\
Irlanda & 0 & 0,0 \\
Italia & 23 & 20,3 \\
Luxemburgo & 0 & 0,0 \\
Portugal & 0 & 0,0 \\
Reino Unido & 20 & 17,7 \\
TOTAL & 113 & 100,0 \\
\hline
\end{tabular}

Fuente: Entrevistas del autor (1996-97). Elab. propia. 
es también importante es la holandesa, a la que pertenecen el 15,9\% de los entrevistados. Los belgas y los daneses tienen poca representación, mientras que no se encontró ningún luxemburgués, portugués, griego o irlandés al frente de ningún negocio.

Resulta interesante comparar la procedencia de los residentes extranjeros que habitan en Cataluña, o en las tres provincias costeras, con la de los empresarios turísticos que han sido entrevistados. Así, el 54,3\% de los residentes extranjeros con origen en algún país comunitario (14.991 de los 27.629, en 1994) proceden de algún país del norte de Europa (DIRECCIÓN GENERAL DE MIGRACIONES, 1996). Sin embargo, la proporción de empresarios noreuropeos entre el total de empresarios entrevistados es mucho mayor. Sólo el 74\% corresponden a Alemania, Francia, Países Bajos y el Reino Unido (excluyendo Francia representan el 56,7\%) (tabla 2). Estas cifras manifiestan, por tanto, que la atracción para el establecimiento de una empresa turística en Cataluña es mucho mayor entre los ciudadanos de países del norte de Europa que entre los procedentes de otros países europeos.

Por otro lado, si comparamos las nacionalidades más representadas entre los turistas extranjeros en Cataluña, con las de los empresarios entrevistados, en líneas generales se aprecian grandes similitudes. Así, los extranjeros europeos con negocios turísticos proceden en su mayoría de países del norte de Europa, al igual que los principales flujos de turismo extranjero y europeo a la región. El caso de Francia es excepcional, ya que si los turistas franceses en Cataluña son los mayoritarios entre todos los europeos $(45,8 \%)$, sólo aparecen en un 17,7\% de los negocios. Sin embargo, aunque los alemanes son el segundo grupo más numeroso entre los turistas $(14,3 \%)$, son los más representados entre los empresarios (23\%). En síntesis, incluyendo a Francia, los turistas noreuropeos representan casi el $79,9 \%$ del turismo extranjero en Cataluña, mientras que los negocios en manos de estos ciudadanos de países del norte de Europa representan el 79,7\%. Estas cifras, por tanto, refuerzan la tesis de que un factor que contribuye al mantenimiento de estas empresas en las costas catalanas es la existencia de una demanda turística consolidada y tradicionalmente ligada a los países noreuropeos.

\section{Motivaciones para el establecimiento del negocio}

Los escasos trabajos realizados sobre los aspectos sociales y culturales que intervienen en la formación y funcionamiento de las empresas de turismo coinciden en dos características similares. La primera apunta a que las motivaciones para la creación de empresas turísticas son siempre muy diversas, y a la importancia de las razones no económicas por parte de los empresarios para la constitución de las mismas. La segunda, que analizaremos en el siguiente apartado, es que la mayoría de los empresarios carecen de experiencias profesionales relevantes o de una formación precisa relacionada con el sector turístico (SHAW y WILLIAMS, 1997).

La tabla 3 recoge las principales razones señaladas por los empresarios entrevistados para poner en funcionamiento su empresa. Aunque el número de razones consideradas es bastante 
Tabla 3. Razones para establecer el negocio en Cataluña (1).

\begin{tabular}{|lcr|}
\hline Razones & Nímero & $\%$ \\
\hline Clima y carácter de la gente mediterránea & 73 & 64,6 \\
Había estado antes de vacaciones en la zona & 42 & 37,1 \\
Esposo(a) o compañero(a) es originalmente de esta zona & 29 & 25,6 \\
Familia o amigos viviendo ya en la zona & 27 & 23,9 \\
Facilidad para encontrar un trabajo (gracias al desarrollo turístico) & 22 & 19,5 \\
Por casualidad & 14 & 12,4 \\
Naturaleza y Paisaje & 11 & 9,7 \\
Nuevas experiencias o necesidad de cambio & 11 & 9,7 \\
Tenía ya una vivienda en esta zona & 10 & 8,8 \\
Cercanía de la frontera francesa & 9 & 8,0 \\
Facilidad para abrir un negocio & 8 & 7,1 \\
Esposo(a) o compañero(a) vino a trabajar & 7 & 6,2 \\
Razones políticas & 7 & 6,2 \\
Menores precios y coste de la vida & 6 & 5,3 \\
Desagrado de su país de origen & 4 & 3,5 \\
Otra/s razón/razones & 18 & 16,0 \\
\hline
\end{tabular}

(1) Puesto que estas respuestas derivan de una pregunta abierta en la que los entrevistados dieron más de una contestación, la suma absoluta de ellas no es 113 ni el porcentaje total es el 100\%.

Fuente: Entrevistas del autor (1996-97). Elab. propia.

amplio, la mayoría se pueden agrupar en unas pocas ya que se refieren a cuestiones muy similares. Teniendo en cuenta que cada empresario dio más de una razón de porqué estableció su negocio en Cataluña, la característica más llamativa es que las cinco más comunes tienen relación con aspectos medioambientales y de tipo personal/familiar. Así, el $64,6 \%$ de las respuestas hacen referencia al clima y a la idiosincrasia mediterránea como principales factores de atracción, al previo conocimiento del lugar por haberlo visitado anteriormente en vacaciones $(37,1 \%)$, o al hecho de tener familia o amigos viviendo ya en la zona $(12,4$ y $11,5 \%$ respectivamente). Las razones de tipo personal son también particularmente importantes ya que el 25,6\% de los empresarios señala que en la localización del negocio influyó que su cónyuge o compañero/a fuera de origen catalán. Las únicas razones de tipo más «profesional» fueron dadas por el 19,5\% de los empresarios, al considerar las posibilidades que el desarrollo del sector turístico les brindaba para establecer un negocio. Finalmente, para el $12,4 \%$ de los entrevistados, la apertura del negocio se debió a la casualidad.

En definitiva, la preferencia residencial por una zona de costa apunta a la gran relación existente entre la creación de estos negocios y el deseo de disfrutar de un determinado escenario físico y de un particular estilo de vida. Al igual que WILLIAMS et alt. (1989b) destacaron para la región inglesa de Cornwall, un porcentaje significante de los entrevistados en Cataluña $(37,1 \%)$ eligieron la zona después de haberla visitado en vacaciones. En concreto, un $83,2 \%$ de ellos había disfrutado previamente de vacaciones en mismo área donde luego establecieron su negocio, mientras que el 75,2\% habían visitado exactamente 
la misma población donde más tarde establecerían el negocio. Por otra parte, la fidelidad de esos futuros empresarios a la misma zona de vacaciones queda reflejada en que el $21,4 \%$ de ellos la habían visitado seis o más veces en los cinco años previos a la instalación del negocio. Parece claro, por tanto, que los destinos de vacaciones se convierten en objetivos potenciales de migración en el futuro. Así, al igual que destacan BULLER y HOGGART (1994) para los británicos que trasladan su residencia a zonas rurales francesas, el previo consumo turístico de la zona actúa como factor de localización posterior, constituyendo una importante razón para el futuro cambio de residencia y de trabajo. Mediante este mecanismo, lo que comienza siendo un traslado turístico de carácter temporal, se convierte posteriormente en una migración de carácter más definitivo.

Por el contrario, es apreciable la escasa importancia que en la toma de decisiones han tenido los factores de tipo económico o profesional. Ninguna de las razones señaladas por los empresarios apunta a ese tipo de motivaciones, por lo que, al igual que GORDON (1991) ha destacado para otros grupos de inmigrantes en Europa, la adopción de un determinado papel empresarial no puede ser considerado una respuesta a una exclusión social o económica concreta (como por ejemplo una situación previa de desempleo), ni tampoco una vía para mitigar una situación económica desfavorable entre este grupo de inmigrantes extranjeros. Dicho de otra forma, las motivaciones para el establecimiento pueden ser muchas y variadas, por supuesto extraeconómicas. De hecho, las consideraciones sobre la interacción social entre productores y consumidores en la industria del turismo evidencian que el desarrollo de la industria turística no es explicable simplemente por cuestiones o factores económicos (URRY, 1990). En este sentido, J. URRY considera necesario examinar un conjunto de factores sociales y culturales, bajo una perspectiva de evolución continua, ya que transforman continuamente las expectativas tanto de los turistas como de los empresarios, en relación con el significado y los efectos de la provisión de servicios. Así, la economía aplicada al turismo, o la economía turística, no puede ser entendida sin tener en cuenta las expectativas socioculturales que intervienen en la diversidad tan compleja de tales servicios. En relación con esta teoría, la escasa importancia de los factores económicos en la formación de las empresas turísticas conduce a pensar que la existencia de estos negocios, en muchas ocasiones como esta, responde casi únicamente a esos otros factores sociales y culturales como son el consumo de una cultura particular, un estilo de vida y un entorno con unas características climáticas determinadas.

\section{Experiencias profesionales previas y capitalización de la empresa}

Teniendo en cuenta los estudios realizados sobre la formación de pequeñas empresas en el sector industrial (secundario) en algunos países europeos, una de las características más habituales es que se establezcan empresas en aquellos sectores en los que el empresario tiene experiencia/s profesional/es previa/s (KEEBLE y WEVER, 1986; KEEBLE et alt., 1992). Pero no siempre, ni tampoco en todos los sectores empresariales, el tipo de empresa que se va a establecer tiene relación con la experiencia acumulada previamente 
por el empresario. En turismo, los trabajos realizados por WILLIAMS et alt. (1989a); WILLIAMS et alt. (1989b) y SHAW y WILLIAMS (1988) sobre la formación de pequeñas empresas turísticas en zonas inglesas, constituyen una clara evidencia de ello. En el caso de los empresarios noreuropeos con negocios en Cataluña, las conclusiones a este respecto parecen ser las mismas.

Considerando también como preparación la experiencia previa, la mayoría de los empresarios entrevistados cuentan con escasa, o con nula preparación o experiencia profesional para el puesto de empresarios turísticos, lo cual demuestra que no siempre esa preparación y/o experiencia es relevante para el tipo de empresa que se va a crear. Por ejemplo, sólo un $38,9 \%$ de los entrevistados declararon que su último trabajo desempeñado antes de la instalación de la actual empresa se llevó a cabo en el sector turístico, bien como propietarios / arrendatarios de un negocio (23,9\%), o simplemente como trabajadores (15\%) (tabla 4). Por el contrario, el 55,7\% de los empresarios (el 5,4\% restante NS/NC) no han tenido ningún tipo de relación con el turismo en su último trabajo desempeñado, ni como propietarios-gerentes, ni como trabajadores. Pero aparte de esas cifras, otro dato importante es que sólo el 46,9\% trabajó alguna vez en turismo a lo largo de sus trayectorias profesionales (bien como empresarios o como trabajadores), mientras que el $53,1 \%$ restante no lo hizo nunca.

Preguntados si habían estado al frente de alguna empresa anteriormente, un $58,3 \%$ de los empresarios contestaron afirmativamente, aunque sólo en el 32,7\% de los casos fue una empresa turística (véase la nota 1 de la tabla 4), mientras que en el 25,6\% restante fue en

Tabla 4. Últimos trabajos desempeñados por los empresarios

\begin{tabular}{|lrr|}
\hline Último trabajo desempeñado & Nímero & $\%$ \\
\hline Propietario o gerente de una empresa turística (1) & 27 & 23,9 \\
Propietario o gerente de una empresa en el sector terciario & & \\
(cualquiera, exceptuando las turísticas) & 17 & 15,0 \\
Propietario o gerente de una empresa en otro sector económico & 3 & 2,6 \\
Trabajador en una empresa turística (2) & 17 & 15,0 \\
Trabajador en el sector terciario (excluyendo empresas turísticas) & 23 & 20,3 \\
Trabajador en otros sectores & 3 & 2,6 \\
Educación / Investigación / Personal sanitario & 2 & 1,8 \\
Sin trabajo previo / Desempleado & 3 & 2,7 \\
Estudiante & 7 & 6,2 \\
Otro trabajo (en el sector terciario o no) & 5 & 4,4 \\
No sabe / No contesta & 6 & 5,3 \\
TOTAL & 113 & 100,0 \\
\hline
\end{tabular}

(1) Se consideran empresasa turísticas las de hosteleria (bares, restaurantes, cafés, pubs, discotecas, etc.) y hotelería-alojamiento (hoteles, hostales, pensiones, etc.).

(2) La consideración de «trabajador» excluye a los propietarios y arrendatarios.

Fuente: Entrevistas del autor (1996-97). Elab. propia. 
otros sectores de la economía. En definitiva, estas cifras demuestran que la vía o el camino más común para el acceso al empresariado turístico es desde puestos desempeñados como empresarios, o bien como simple trabajadores en el sector (46,9\% de los casos). A juzgar por el $53,1 \%$ restante, se puede afirmar que esos empresarios acceden a estas empresas sin haber desempeñado nunca ningún empleo en el sector.

Al igual que han mostrado otros estudios sobre el empresariado turístico (SHAW y WILLIAMS, 1987), la mayoría de estos pequeños empresarios de turismo en Cataluña cuentan con una escasa preparación profesional. Partiendo de la base de que para el desempeño de este trabajo no se requiere ninguna cualificación ni preparación especial (de forma obligada), la falta de preparación formal (empresarial) no parece constituir una barrera importante para establecer una empresa de turismo. Sólo el 8,8\% de los empresarios cuentan con algún tipo de cualificación o preparación universitaria, lo cual no quiere decir que sea la requerida o idónea para ser empresario turístico. Sin embargo, la cualificación académica es sólo una parte de la "educación» empresarial. En este sentido, la mayoría de los empresarios han desarrollado su faceta empresarial desde la práctica y la experiencia adquiridas, bien como empresarios o como empleados de otros negocios anteriores. En definitiva, este bajo nivel de preparación es consecuencia de las escasas barreras existentes para entrar en la industria turística, y en este caso para establecer un pequeño negocio turístico.

Otro de los aspectos que nos indica las condiciones en que operan estas empresas y nos revela alguna característica de los inmigrantes a su frente es el de su capitalización. En principio, el acceso limitado a fuentes de financiación es una de las características comunes en la formación de pequeñas y medianas empresas del sector turístico en todos los países europeos. No siendo así para las grandes empresas, este hecho constituye un grave problema de cara a la inversión y desarrollo de las mismas (EUROPEAN COMMISSION, 1997). La tabla 5 muestra para nuestro caso cómo los ahorros personales constituyen la principal fuente de financiación utilizada por los empresarios entrevistados (para el $64,6 \%$ ), aunque no de forma exclusiva, sino compartida con otro/s tipo/s de financiación. El número de empresarios que únicamente dependieron de los ahorros personales para establecer su empresa fue $33(29,2 \%)$, mientras que el otro $35,4 \%$ utilizó esta fuente con

Tabla 5. Financiación de la empresa

\begin{tabular}{|lcc|}
\hline Fuente de financiación & Nimero & $\%$ \\
\hline Ahorros personales & 73 & 64,6 \\
Préstamo o crédito bancario & 46 & 40,7 \\
Venta de alguna propiedad & 18 & 15,9 \\
Otro préstamo o crédito & 8 & 7,1 \\
Otra forma de financiación & 7 & 6,2 \\
Préstamo familiar & 6 & 5,3 \\
\hline
\end{tabular}

Fuente: Entrevistas del autor (1996-97). Elab. propia. 
alguna otra. En segundo lugar, los préstamos o créditos bancarios fue la segunda fuente más utilizada ( $40,7 \%$ de los entrevistados) y, por orden de importancia, también la venta de alguna propiedad particular (15,9\%).

Este alto porcentaje de empresarios dependientes de ahorros personales como vía de capitalización empresarial nos lleva a una consideración de interés. Según SCHWANINGER (1986), el predominio de esta vía de financiación «informal» y no comercial es característico de un tipo de empresas controladas por personas con una concepción muy limitada de la necesidad de establecer estrategias empresariales o planes de empresa. De alguna manera, ese tipo de financiación condiciona, o es reflejo también, del tipo de inversiones que se llevarán a cabo, en el sentido de que una escasa capitalización limita las inversiones posteriores. Así, pequeñas inversiones como las que estos empresarios llevan a cabo, son perfectamente acometibles con sus ahorros personales, procedentes muchas veces de antiguos trabajos en sus países de origen. En definitiva, el uso mayoritario de ahorros particulares tiende a reforzar la idea de la naturaleza casual de estas empresas, así como su escasa estabilidad financiera.

\section{CONCLUSIONES}

Las migraciones masivas producidas entre los países del sur y del norte de Europa durante los años 60 y 70, en su mayoría originadas por motivos económicos, han sido sustituidas por nuevas formas de movilidad, algunas de las cuales poco tienen que ver con causas estrictamente laborales (OECD, 1992). Uno de los nuevos tipos de desplazamiento es el de personas de países del centro y norte de Europa hacia países europeos meridionales. Es el caso de las migraciones internacionales de población retirada (WILLIAMS et alt., 1997) $u$ otros tipos de migraciones que, aunque de población económicamente activa, están motivadas fundamentalmente por causas extraeconómicas. En este artículo hemos analizado un tipo concreto de movimientos como es el de los inmigrantes de países comunitarios que se trasladan a las costas de Cataluña para establecer pequeños negocios turísticos. De esta forma, se contribuye al estudio de unos flujos de población a los que se les ha prestado escasa atención dentro de los dedicados a las migraciones internacionales en Europa.

El resultado del análisis de este tipo de inmigrantes económicamente activos en una zona turística nos permite identificar las fuertes limitaciones que ofrecen las teorías sobre migraciones internacionales para explicar este tipo concreto de desplazamientos internacionales dentro de Europa (LARDIÉS, 1999). La aplicación, por tanto, de las principales teorías sobre migraciones internacionales a estos desplazamientos, en su mayor parte motivados por razones no económicas, no ofrece, en principio, resultados satisfactorios para la explicación de las acciones de este tipo de inmigrantes. Detrás del cambio de residencia que conlleva la emigración, en muy pocos casos las razones de tipo laboral o profesional han incitado directamente a la emigración, a pesar de que la mayoría 
de la población entrevistada es económicamente activa y los inmigrantes están al frente de negocios turísticos. Aunque todos ellos obtienen sus ingresos en Cataluña, en muy pocos inmigrantes se han detectado preferencias de tipo profesional para elegir esta región como destino residencial y localizar en ella un negocio; al contrario, los criterios de localización han estado marcados por motivos como la atracción del entorno, las experiencias vacacionales previas o razones familiares como que el cónyuge-compañero/a sea originario/a de la región o del mismo núcleo. De hecho, las entrevistas llevadas a cabo con estos inmigrantes-empresarios han puesto de relevancia que la decisión de establecer un negocio casi siempre es posterior a la de emigrar, por lo que la participación en el sector turístico es, casi siempre, un hecho secundario y depende, por ser una decisión tomada posteriormente, de la decisión de emigrar y de los condicionantes o razones personales y familiares.

El análisis de las motivaciones muestra cómo el clima, las condiciones ambientales, la tranquilidad, o el estilo de vida, son dominantes en la decisión de emigrar, aunque también las razones familiares y la atracción de los destinos costeros por su desarrollo turístico (tabla 3); ese último factor, por contra, es el único que permite considerar ciertas motivaciones «económicas» en el traslado, si por motivación económica puede entenderse la posibilidad que ofrece para la instalación de un negocio de este tipo el desarrollo turístico del área y el cierto "ambiente empresarial» que tal desarrollo ofrece. Aparte de que las variables económicas no influyan prácticamente en el futuro lugar de residencia y de localización de la empresa, el bajo nivel de experiencia previa en el sector (tabla 4), la falta de preparación en turismo y las limitaciones de estas empresas a fuentes comerciales de capitalización (tabla 5) son variables que definen el perfil de estos inmigrantes y sus empresas.

El análisis de otros factores (no analizados en este artículo) como la importancia de la participación familiar en el negocio, la escasa creación de empleo extra-familiar, o algunas otras prácticas relacionadas con el funcionamiento de estas empresas, muestran que las cuestiones económicas generalmente aparecen en un segundo plano, no siendo verdaderamente el «motor» del negocio; más bien, las empresas son la llave para que el empresario pueda seguir disponiendo de una determinada calidad de vida que él mismo se ha marcado, más que una vía o herramienta para la expansión económica del negocio o la generación de nuevo empleo. En consecuencia, más que estar «sometidas» estas empresas a una optimización económica, lo están a unos dictados marcados por el disfrute de unas determinadas condiciones y estilos de vida por parte de sus propietarios-gerentes.

Al igual que se ha sugerido al estudiar el caso de algunas migraciones internacionales de población retirada, si las acciones relativas a la emigración de los ciudadanos extranjeros a las costas catalanas no son explicadas desde el punto de vista teórico por los modelos sobre migraciones internacionales, cabe la posibilidad de que sean explicados como si de una extensión de las migraciones intranacionales se trataran; en tal caso, estas migraciones se asemejarían a las desencadenadas por el fenómeno de la contraurbanización, desde zonas urbanas a rurales, aunque sin rebasar las fronteras nacionales. Eso han sugerido, por ejemplo, BULLER y HOGGART (1994) para explicar los nuevos asentamientos de 
británicos en zonas rurales francesas. Desde este punto de vista, las migraciones de británicos a Francia tienen muchos elementos en común con las de ciudadanos comunitarios a las costas catalanas. En ambos casos, los emigrantes buscan lugares con baja urbanización y relativamente baratos, tranquilidad y con determinadas condiciones ambientales, por lo que la calidad de vida se convierte en un elemento esencial para decidir el nuevo lugar de residencia. Eso explicaría, en parte, la selección de un lugar mediterráneo como lugar para establecer un negocio de estas características. Sin embargo, aparte de buscar un paisaje costero y un estilo de vida diferente, la contraurbanización es algo más. Supone un deseo de alternativa, alejado de formas de vida típicamente urbanas. En este sentido, analizando las motivaciones que empujan al traslado de este tipo de emigrantes, junto con las características de las zonas de recepción, la contraurbanización provee algunas respuestas, aunque no completamente satisfactorias, para explicar algunos aspectos geográficos relacionados con las migraciones de población económicamente activa desde países comunitarios a destinos mediterráneos.

\section{BIBLIOGRAFÍA}

ANTON CLAVÉ, S., LÓPEZ PALOMEQUE, F., MARCHENA GÓMEZ, M.J. y VERA REBOLLO, J.F. (1996): «La investigación turística en España: Aportaciones de la geografía». Estudios Turísticos, $129,165-208$.

BULLER, H. y HOGGART, K. (1994): International counterurbanization: British migrants in rural France. Ed. Avebury. Aldershot.

DIRECCIÓN GENERAL DE MIGRACIONES (1996): Anuario de Migraciones, 1995. Ed. Dirección General de Migraciones, Ministerio de Asuntos Sociales. Madrid.

EUROPEAN COMMISSION (1997): Yield management in small and medium-sized enterprises in the tourism industry. General report. Ed. Office for Official Publications of the European Communities. Luxemburgo.

GIBSON, I. (1992): Fire in the Blood: The Nezo Spain. Ed. Faber. Londres.

GORDON, I. (1991): The impact of economic change on minorities and migrants in Western Europe. Reading, Discussion Paper, 2, Department of Geography, University of Reading. Reading.

IEC (1992): Censo de la Población Española, 1991. Instituto de Estadistica de Cataluña. Barcelona.

KEEBLE, D. y WEVER, E. (1986): «Introduction». En: KEEBLE, D. y WEVER, E. (Eds.): New firnts and regional development in Europe. 1-34. Crom Hell. Dover.

KEEBLE, D.; BRYSON, J. y WOOD, P. (1992): «Entrepreneurship and flexibility in business services: the rise of small management consultancy and market research firms in the UK». En: CALEY, 
K.; CHELL, E.; CHITTENDEN, F. y MASON, C. (Eds.): Snall enterprise development. Policy and practice in action. 43-58. Paul Chapman Publishing Ltd. Londres.

KING, R. y RYBACZUK, K. (1993): «Southern Europe and the international divison of labour: from emigration to immigration». En: KING, R. (Ed.): The New Geography of European Migrations . 175-206. Belhaven. Londres.

LARDIÉS, R. y HOGGART, K. (1996): «Immigrants into Spain from the European Union». Artículo presentado a la MEDITERRANEAN CONFERENCE ON POPULATION, MIGRATION AND DEVELOPMENT organizada por el Consejo de Europa, Palma de Mallorca, 15-17 Octubre 1996 (15 pp.).

LARDIÉS, R. (1999): «Migration and tourism entrepreneurship: north-European immigrants in Cataluña and Languedoc», International Joutnal of Population Geography. 5, 477-491.

MONFORT MIR, M.; MORANT MORA, A. e IVARS BAIDAL, J. (1996): «Demanda turística». En: PEDREÑO MUNOZ, A. (Dir.) y MONFORT MIR, V.M. (Coord.): Intraducción a la Economía del Turismo en España. 69-101. Civitas. Madrid.

NAYLON, J. (1992): «Ascent and Decline in the Spanish Regional System». Geography. 77, 334, parte 1, January, 46-62.

OECD (1992): Trends in International Migration. SOPEMI. Ed. Organization for Economic Cooperation and Development. París.

OECD (1994): Trends in International Migration. SOPEMI. Ed. Organization for Economic Cooperation and Development. París.

SALT, J. (1996): «Migration pressures on Western Europe». En: COLEMAN, D. (Ed.): Europe's Population in the 1990s. 92-126. Oxford University Press. New York.

SCHWANINGER, M. (1986): «Strategic business management in tourism». Tourism Management. 7, 2, 77-85.

SECRETARÍA GENERAL DE TURISMO (1995): Nota de Coyzintura Turística.. Enero. Ed. Secretaría General de Turismo, Ministerio de Comercio y Turismo. Madrid.

SHAW, G. y WILLIAMS, A.M. (1987): «Firm formation and operating characteristics in the Cornish tourist industry the case of Looe». Tourism Management. 8, 4, 344-348.

SHAW, G. y WILLIAMS, A.M. (1988): «Tourism and employment: reflections on a pilot study of Looe, Cornwall». Area. 20, 1, 23-34.

SHAW, G. y WILLIAMS, A.M. (1990): «Tourism, economic development and the role of entrepreneurial activity». En: COOPER, C.P. (Ed.): Progress in Tourism, Recreation and Hospitality Management.. Vol. 2. 67-81. John Wiley and Sons. Chischester.

SHAW, G. y WILLIAMS, A.M. (1997): «The private sector: tourism entrepreneurship -a constraint or resource?» En: SHAW; G. y WILLIAMS, A.M. (Eds.): The rise and fall of British constal resorts: cultural and econonic perspectives. 117-136. Pinter. Londres. 
SHAW, G. y WILLIAMS, A.M. (1998): «Entrepreneurship, small business culture and tourism development». En: IOANNIDES, D. y DEBBAGE, K.C. (Eds.): The Tourist Industries: A geographical Analysis. 337-369. Routledge. Londres.

SIMON, G. (1993): «Internal migration and mobility». En: NOIN, D. y WOODS, R. (Eds.): The Changing Population of Europe. 170-184. Blackwell. Oxford.

URRY, J. (1990): The Tourism Gaze Leisure and Travel in Contemporary Societies. Ed. Sage. Londres.

WALDINGER, R.; ALDRICH, H.; WARD, R. et alt. (1990): Ethnic Entrepreneurs. Immigrant Business in Industrial Societies. Ed. Sage. Newbury Park.

WARD, R. (1991): «Economic development and ethnic business». En: CURRAN, J. y BLACKBURN, R.A. (Eds.): Paths of enterprise. The future of the small business. 51-67. Routledge. Londres.

WILLIAMS, A.M.; KING, R. y WARNES, T. (1997): «A place in the sun: international retirement migration from northern to southern Europe». European Urban and Regional Studies. 2, 4, 115-134.

WILLIAMS, A.M. y SHAW, G. (1988): «Tourism and development: introduction». En: WILLIAMS, A.M. y SHAW, G. (Eds.): Tourism and Economic Development.. 1-11. Belhaven. Londres.

WILLIAMS, A.M.; GREENWOOD, J. y SHAW, G. (1989a): Tourism in the Isles of Scilly: A study of small firms on small islands. Tourism Research Group, Department of Geography, University of Exeter. Exeter.

WILLIAMS, A.M.; SHAW, G. y GREENWOOD, J. (1989b): «From tourist to tourism entrepreneur, from consumption to production: evidence from Cornwall, England». Environment and Planning. A21, 1639-1653.

ZLOTNIK, H. (1992): «Empirical identification of international migration systems». En: KRITZ, M.M.; LIM, L.L. y ZLOTNIK, H. (Eds.): International Migration Systems. 19-40. Clarendon. Oxford. 